\title{
Organism Profile in Periprosthetic Joint Infection: Pathogens Differ at Two Arthroplasty Infection Referral Centers in Europe and in the United States
}

\author{
Vinay K. Aggarwal, MD ${ }^{1}$ Hooman Bakhshi, MD ${ }^{1} \quad$ Niklas Unter Ecker, MD ${ }^{2}$ Javad Parvizi, MD, FRCS ${ }^{1}$ \\ Thorsten Gehrke, MD² Daniel Kendoff, MD, $\mathrm{PhD}^{2}$
}

${ }^{1}$ Department of Orthopaedic Surgery, Rothman Institute, Philadelphia, Pennsylvania

2 Department of Orthopaedic Surgery, HELIOS ENDO-Klinik Hamburg, Hamburg, Germany

\begin{abstract}
Address for correspondence Daniel Kendoff, MD, PhD, Department of Orthopaedic Surgery, HELIOS ENDO-Klinik Hamburg, Holstenstrasse 2, Hamburg 22767, Germany (e-mail: daniel.kendoff@helios-kliniken.de; vinayagg1@gmail.com).
\end{abstract}

J Knee Surg 2014;27:399-406.

\begin{abstract}
Keywords

- periprosthetic joint infection

- PJI

- arthroplasty

- Europe

- United States

Infecting microorganism is a strong predictor of treatment success for periprosthetic joint infection (PJI). The purpose of this study was to compare the infecting pathogens causing PJI at two large infection referral centers in the United States and in Europe. In this study, 898 consecutive cases of PJI were identified at the HELIOS ENDO-Klinik Hamburg in Europe and 772 cases were identified at the Rothman Institute in the United States. The incidence of organisms at the HELIOS ENDO-Klinik Hamburg versus the Rothman Institute was: coagulase-negative Staphylococcus (39.3 vs. 20.2\%), S. aureus (13.0 vs. $31.0 \%$ ), Streptococcus (6.5 vs. $5.8 \%$ ), Enterococcus (7.0 vs. $3.9 \%$ ), anaerobic (9.0 vs. $0.9 \%$ ), fungal ( 0.3 vs. $2.3 \%$ ), mycobacterial ( 0 vs. $0.6 \%$ ), polymicrobial (3.4 vs. $7.4 \%$ ), culture negative ( 16.1 vs. $15.8 \%$ ), and other organisms ( 0.9 vs. $5.4 \%$ ). The percentage of methicillin-resistant $\mathrm{S}$. aureus was significantly higher at the American center than at the European center ( 48.1 vs. $12.8 \% ; p<0.0001)$. Our findings show higher virulence and resistance organisms are more prevalent at a referral center in the United States compared with one in Europe.
\end{abstract}

It is well known by the health care community that periprosthetic joint infection (PJI) is one of the most devastating complications following total joint arthroplasty (TJA). Although the overall incidence of PJI is relatively low, estimated at 0.5 to $2 \%$ following total hip arthroplasty (THA) and at 1 to $3 \%$ following total knee arthroplasty (TKA), ${ }^{1-4}$ an immense economic burden is associated with PJI. ${ }^{5-8}$ On the basis of a projection study, around $\$ 650$ million was spent in the United States alone to treat PJI last year with the burden expected to grow rapidly over the next few years unless the trend can be reversed. ${ }^{9}$

Preoperative antibiotic prophylaxis and empirical antibiotic treatment are often dependent on the organism responsible for PJI. ${ }^{10}$ Furthermore, several studies have shown that treatment outcomes for eradication of PJI vary widely depending on

received

November 7, 2013

accepted

November 19, 2013

published online

January 10, 2014

infecting pathogen. ${ }^{11-15}$ For example, a study by Zmistowski et al in 2011 showed only a 52\% success rate for treatment of gram-negative PJI with two-stage exchange arthroplasty. ${ }^{8}$ In addition, methicillin-resistant Staphylococcus aureus (MRSA) infection is particularly difficult to treat and serves as an independent risk factor for treatment failure. ${ }^{8,11}$ Therefore, to guide effective perioperative antibiotic prophylaxis, empirical antimicrobial therapy, and optimal surgical management, it is very important to recognize the microorganism profile responsible for PJI. ${ }^{10,16,17}$

Although the overall incidence of infecting organisms in PJI has remained somewhat stable over the years and across the globe, small changes in bacterial resistance patterns may have a profound impact on treatment algorithms in the future. ${ }^{18-20}$

Copyright $\odot 2014$ by Thieme Medical Publishers, Inc., 333 Seventh Avenue, New York, NY 10001, USA. Tel: +1(212) 584-4662.
DOI http://dx.doi.org/ 10.1055/s-0033-1364102. ISSN 1538-8506. 
Unfortunately, the incidence of MRSA-related PJI in the United States has increased in the past several years. ${ }^{6}$ In small part, this finding has led to adoption of two-stage exchange arthroplasty as the preferred definitive treatment for infection eradication in this country. ${ }^{21}$ Meanwhile, onestage direct exchange procedures are used more heavily in European versus American institutions with authors citing a reduced cost and presumed ease for the patient as justification for the use of these direct exchanges. ${ }^{22}$

To this point, although there have been studies comparing postsurgical treatment outcomes after PJI between the United States and Europe, there is no literature evaluating the organisms responsible for infection after TJA in these two regions. The goal of this study is to provide a direct comparison of the infecting pathogens causing PJI in both hips and knees at two high-volume tertiary infection referral centers in the United States and in Europe.

\section{Methods}

We performed a retrospective review of all intraoperative intra-articular tissue cultures taken at time of joint revision for infection from patients at two high-volume infection referral centers between 2000 and 2011. At a European orthopedic surgery center, the HELIOS ENDO-Klinik Hamburg, Germany, 898 cases of PJI ( 568 hips and 330 knees) were identified. At an American orthopedic surgery center, the Rothman Institute in Philadelphia, Pennsylvania, 772 cases of PJI (353 hips and 419 knees) were identified. Infected cases were identified using each institution's prospective electronic joint infection database. PJI was defined according to the clinical and laboratory parameters: (1) painful or swollen joint with or without draining sinus tract; (2) positive joint aspirate cultures; (3) elevated serum erythrocyte sedimentation rate $(>30 \mathrm{~mm} / \mathrm{h})$ and C-reactive protein $(>1.0 \mathrm{mg} / \mathrm{dL})$ markers; (4) elevated white synovial white blood cell count and polymorphonuclear cell differentiation; and (5) purulence encountered intraoperatively.

Information obtained from electronic medical records included age, gender, body mass index (BMI), index joint, date of culture, culture source and medium, intraoperative culture results, duration of culture, and antibiotic sensitivity of infecting organism as tested with the standard protocol of each institution's microbiology laboratory (not all nonstaphylococcal bacteria were tested for susceptibility to every possible antibiotic). Date of index operation, operative reports from index procedure (therefore distinction between infected primary or revision), and history of preadmission antibiotic therapy were not available for all cases as both institutions serve as an infection referral center for outside hospitals. Therefore, due to the fact that $>75 \%$ of infections treated were referrals, subgroup analyses for these variables were not possible.

At both institutions, patients are required to take a shower the evening before. At the Rothman Institute, patients were given a prescription for and encouraged to shower with Hibiclens wash with $4 \% \mathrm{w} / \mathrm{v}$ chlorhexidine gluconate (Mölnlycke Health Care US, LLC, Norcross, GA). At the HELIOS ENDOKlinik Hamburg, shaving of incision site with clippers or razor is done in every case the day before surgery, whereas this was not routine at the Rothman Institute. No admission preoperative decolonization of nares for MRSA was undertaken routinely at each institution. In the operating room, patients' skin was prepared with isopropyl alcohol only at the HELIOS ENDO-Klinik Hamburg, whereas a variety of surgical preparation combinations including $2 \%$ chlorhexidine gluconate/ $70 \%$ isopropyl alcohol, $0.7 \%$ iodine povacrylex/74\% isopropyl alcohol, and/or povidone iodine were used at the Rothman Institute. Perioperative antibiotics were delayed until after culture obtainment at both institutions for infected revision surgeries during the study period. During the primary arthroplasties, intravenous (IV) cefazolin was given 30 minutes before incision at the HELIOS ENDO-Klinik Hamburg, whereas IV cefazolin, clindamycin, or vancomycin was given within 60 minutes of incision at the Rothman Institute.

A minimum of three specimens were taken from each joint and sent for aerobic/anaerobic, fungal, and acid-fast bacilli culture growth. Tissues were homogenized and standard cultivation media was used to grow each type of culture. No special culture techniques such as implant sonication or PCR were used by either center. Cultures were all incubated in appropriate conditions for a minimum of 5 days. Mean duration of culture growth was 14 days at the HELIOS ENDO-Klinik Hamburg and 6.8 days at the Rothman Institute. All infections considered for the study were deep PJIs, not superficial incisional or wound infections. No data on timing of infection were available for categorization in this study; however, all possible PJIs were included from both centers, thus there was no exclusion based on timing of symptoms.

A culture was considered monomicrobial when all cultures from a given case were in agreement for both infecting organism and antibiotic susceptibility profile. A culture was considered polymicrobial when two or more unique infecting organisms were present in either a single specimen or multiple specimens from a single patient case. Culture results were then grouped according to individual species type, gram-positive versus gram-negative organism, anaerobic organisms, monomicrobial versus polymicrobial infection, and based on resistance to antibiotic sensitivity testing. All coagulase-negative staphylococcal isolates were differentiated and categorized together. Oxacillin resistance was used to classify staphylococcal organisms as resistant to methicillin.

For statistical analysis, means and frequencies were used for continuous and categorical variables, respectively. The Fisher exact test was used to compare the regional variances of the infecting organisms as well as differences between infecting organisms in the hip or knee within a particular region. Statistical significance was determined using the Bonferroni correction for multiple bivariate tests done on the same set of data. Initial statistical significance was set at $p=0.05$ and corrected based on $n$ number of Fisher exact tests run on data, such that the ultimate significance was $p=0.05 / n$.

\section{Results}

When comparing the 772 cases of infection at the Rothman Institute to the 898 cases of infection at the HELIOS ENDO- 
Klinik Hamburg, the mean age of patients was 66.19 and 68.14 years, respectively $(p=0.001)$. Males constituted 50.8 and $50.1 \%$ of cases at the Rothman Institute and the HELIOS ENDO-Klinik Hamburg, respectively $(p=0.798)$. The mean BMI was significantly greater in the patients treated at the Rothman Institute compared with the patients from the HELIOS ENDO-Klinik Hamburg (31.9 vs. $28.2 \mathrm{~kg} / \mathrm{m}^{2}$, respectively; $p<0.0001$ ).

The overall incidence of infecting organism at the American and European centers is shown in - Fig. 1. More than 50\% of PJIs in both the United States and European centers were caused by staphylococcal organisms. The incidence of $S$. aureus infections was significantly greater in the United States center than in the European center $(p<0.0001$, odds ratio [OR], 2.99; 95\% confidence interval [CI], 2.32-3.87). Likewise, the incidence of coagulase-negative staphylococcal infections was significantly greater in the European center $(p<.0001$, OR $2.11 ; 95 \%$ CI 1.68-2.66). After statistical correction for multiple bivariate analyses, polymicrobial $(\mathrm{p}=.0002)$ and anaerobic $(\mathrm{p}=.0001)$ species also showed significant difference between the two centers with regards to infecting species.

As part of the "other" bacteria reported, the Rothman Institute identified infections caused by Micrococcus, Bacillus, Corynebacterium, Clostridum, and Coryneform species. The HELIOS ENDO-Klinik Hamburg found infections caused by Corynebacterium species and Listeria monocytogenes. The European center demonstrated a greater variety of coagulase-negative staphylococcal and Streptococcal species including Staphylococcus capitis, Staphylococcus caprae, Staphylococcus lugdunensis, Streptococcus agalactiae, and Streptococcus salivarius, among others.

When analyzing the data with regard to methicillin resistance, there was a significantly greater incidence of resistant Staphylococcal species overall in the United States center compared with the Europe center (49.6 vs. 37.0\%; $p=0.0002$ ). The incidence of MRSA was significantly greater in the United States center than in the European center $(p<0.0001$, OR, 6.27; 95\% CI, 3.39-12.31) (-Fig. 2). The incidence of methicillin-resistant coagulase-negative Staphylococcus was greater in the United States cohort but this difference did not reach statistical significance $(p=0.091)$ (-Fig. 2). The yearly incidences of MRSA and methicillinresistant staphylococcal infections as a percentage of all PJIs at the United States center and European center are shown in -Figs. 3 and 4. Using regression analysis, there was no significant correlation with time within two centers. Further, 8/30 (26.7\%) of the cases of Enterococcus in the United States center were vancomycin resistant, whereas $0 / 63(0.0 \%)$ cases of Enterococcus at the European center were resistant.

After breaking down the cases of infection by joint type, only culture negative infections within the European center showed a significant difference between knees and hips ( 25.2 vs. $10.9 \%$, respectively; $p<0.0001$ ). There were no other significant differences in infecting organisms when comparing knees to hips within either institution (- Table $\mathbf{1}$ ).

\section{Discussion}

Deep joint infection after TJA was characterized by Charnley and Eftekhar in their 1969 study where they account that greater than $50 \%$ of their infections were due to S. aureus (no methicillin resistance reported in any cases), 20\% were culture negative, and the rest were due to Bacillus proteus, Staphylococcus albus, and Streptococcus agalactiae. ${ }^{23}$ Many studies since then have described the bacterial incidence in isolated series of PJIs; however, there have been no studies comparing the infecting organisms between international groups. This study addressed the potential differences in organism profile in PJI of both THA and TKA between two infection referral centers in the United States and Europe.

The current study, which examined cultures from two regions between 2000 and 2011, concurred with organism profiles from previous studies done in either the United States or Europe. The studies from the United States by Fitzgerald, Fulkerson et al, and Schinsky et al show that an increasing percentage of PJIs in American centers are due to S. aureus (-Table 2). ${ }^{18,19,24}$ Meanwhile, studies from long-term

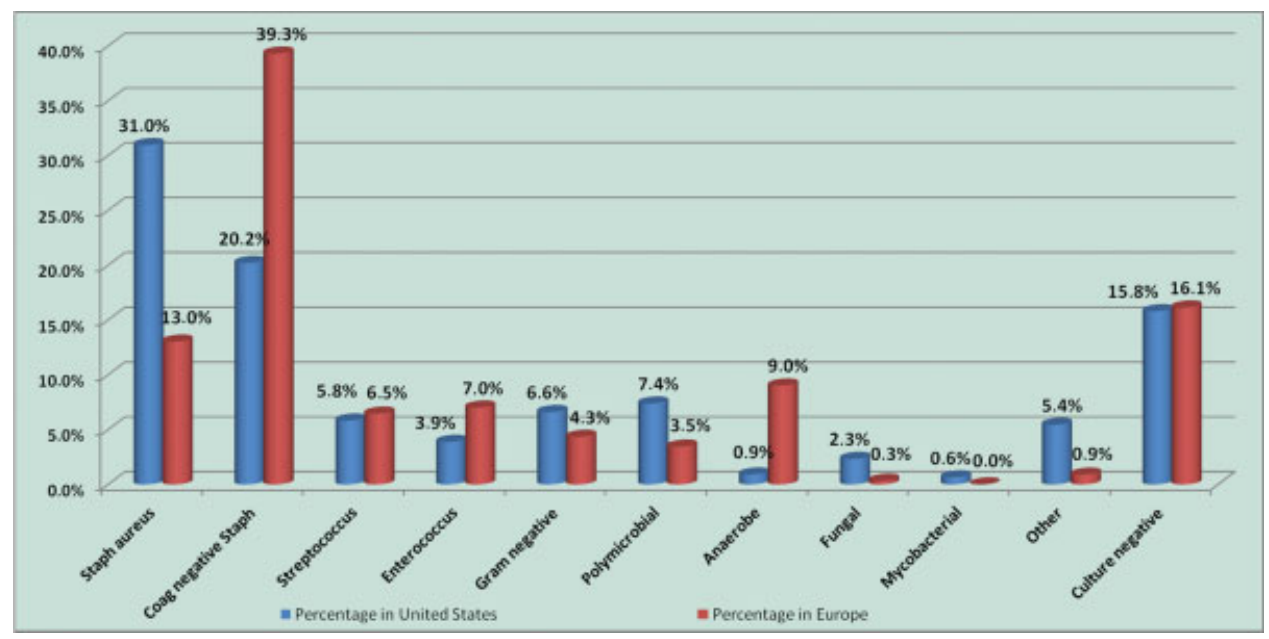

Fig. 1 Organism profile difference in incidence between the Rothman Institute (United States) and Endo-Klinik (Europe). 


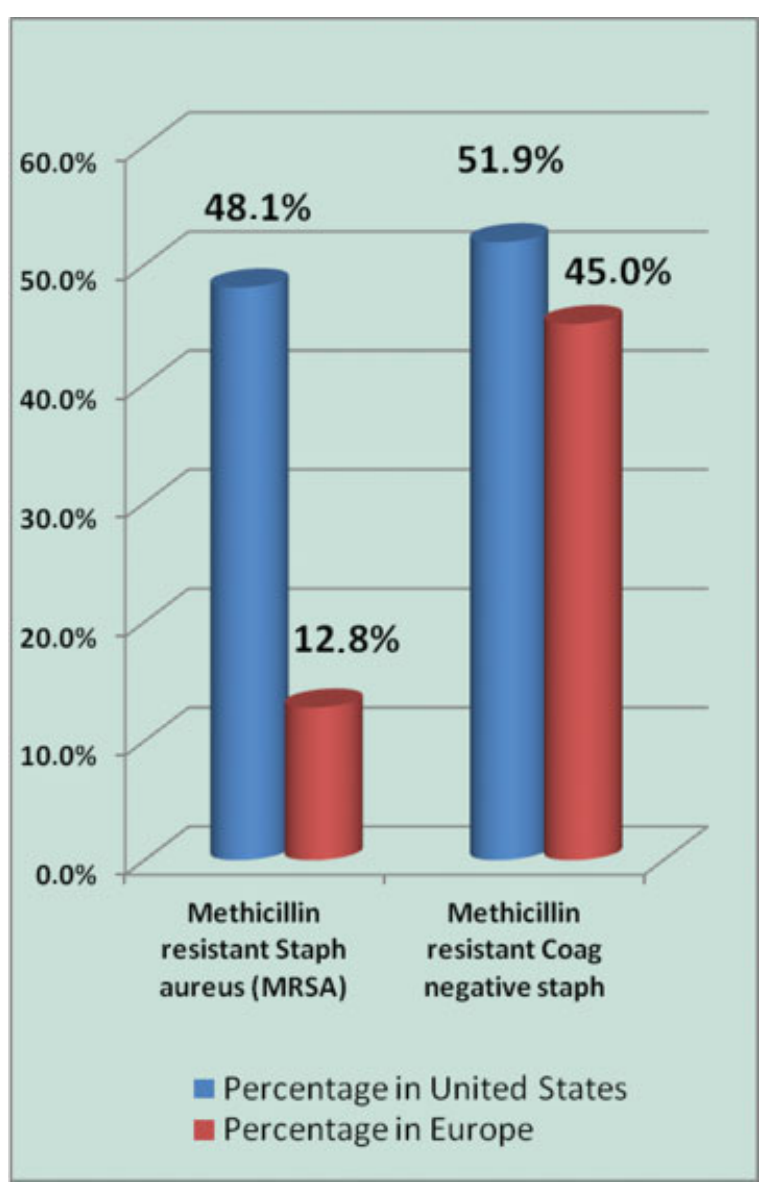

Fig. 2 Methicillin resistance profiles of staphylococcal species in Europe and United States.

prospective European registries including Stefánsdóttir et al in Sweden and Phillips et al in the United Kingdom show a greater contribution of coagulase-negative Staphylococcus and Streptococcus species when compared with $S$. aureus (-Table 2). ${ }^{3,25}$ Both the current study and those studies previously mentioned show that, on average, a greater pro- portion of PJIs in European centers may be due to lower virulence organisms such as coagulase-negative staphylococcal and Streptococcus species (non-group A S. pyogenes species) compared with PJIs reported in American centers.

Importantly, our study demonstrates an increased proportion of methicillin-resistant organisms responsible for PJI at the American center compared with the European center. We found $48.1 \%$ of $S$. aureus infections to be due to MRSA at the Rothman Institute, whereas just $12.8 \%$ of $S$. aureus was due to MRSA at the HELIOS ENDO-Klinik Hamburg. Only one study about PJI by Phillips et al from a specialist orthopedic hospital in the United Kingdom reports on antibiotic susceptibility of S. aureus species for literature comparison: the authors account that only $13.6 \%$ of $S$. aureus infections were due to MRSA. $^{3}$ The higher prevalence of antimicrobial-resistant organisms in the United States has been reported previously through the MRSA nasal carriage rates in healthy adult populations, exceeding $1.5 \%$, and increasing from 2000 to 2005. ${ }^{26,27}$ The European Center for Disease Prevention and Control reports substantial variability in methicillin resistance of staphylococcal species between countries in the continent. However, the overall prevalence of resistance is still lower than in the United States, with Scandinavian nations such as Sweden and the Netherlands showing extremely low resistance and MRSA nasal colonization rates of 0 and $0.11 \%$, respectively. ${ }^{28-30}$

There may be several potential reasons for the difference in methicillin resistance characteristics seen in the two institutions in our study, including host factors and hospital infection control practices. Although we lacked sufficient data to compare comorbidity indices between patients at the two institutions in our study, we found a significant difference in BMI (31.9 vs. $28.2 \mathrm{~kg} / \mathrm{m}^{2} ; p<0.0001$ ). Several reports have questioned whether increased obesity may in fact lead to higher rates of antimicrobial resistance, specifically due to inadequate antibiotic dosing in these larger patients. ${ }^{31,32}$ Further studies will be needed to definitively correlate dosing of antibiotics in obese patients with the resulting antibiotic resistance of infecting organisms in PJI. However, an initial

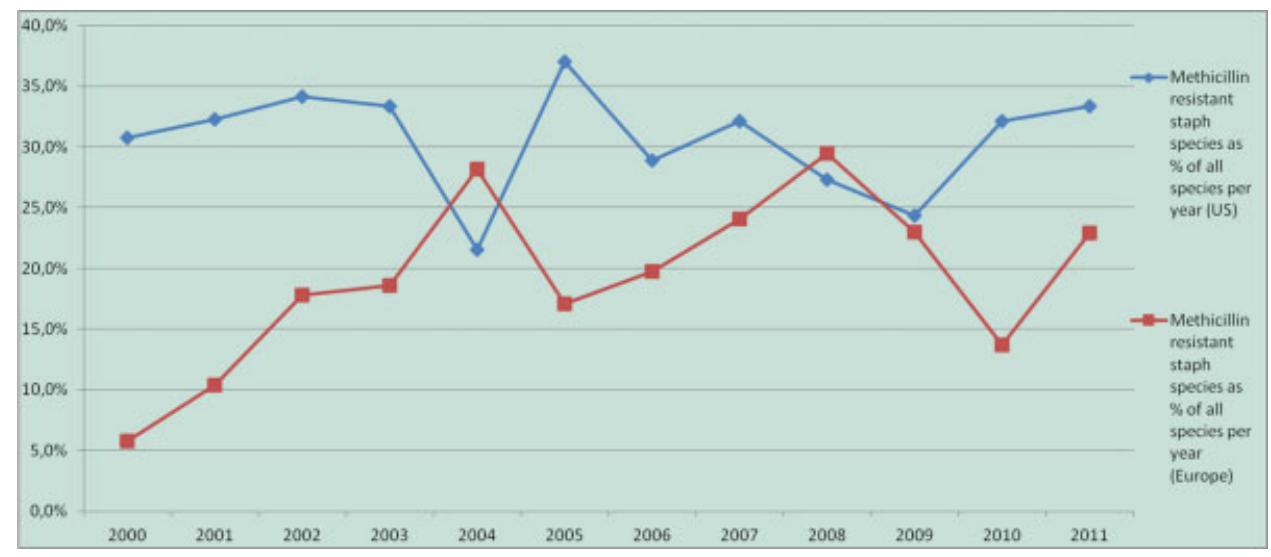

Fig. 3 Yearly trends of methicillin resistance staphylococcal species (as percentage of total PJIs in given year) between U.S. center and European center. 


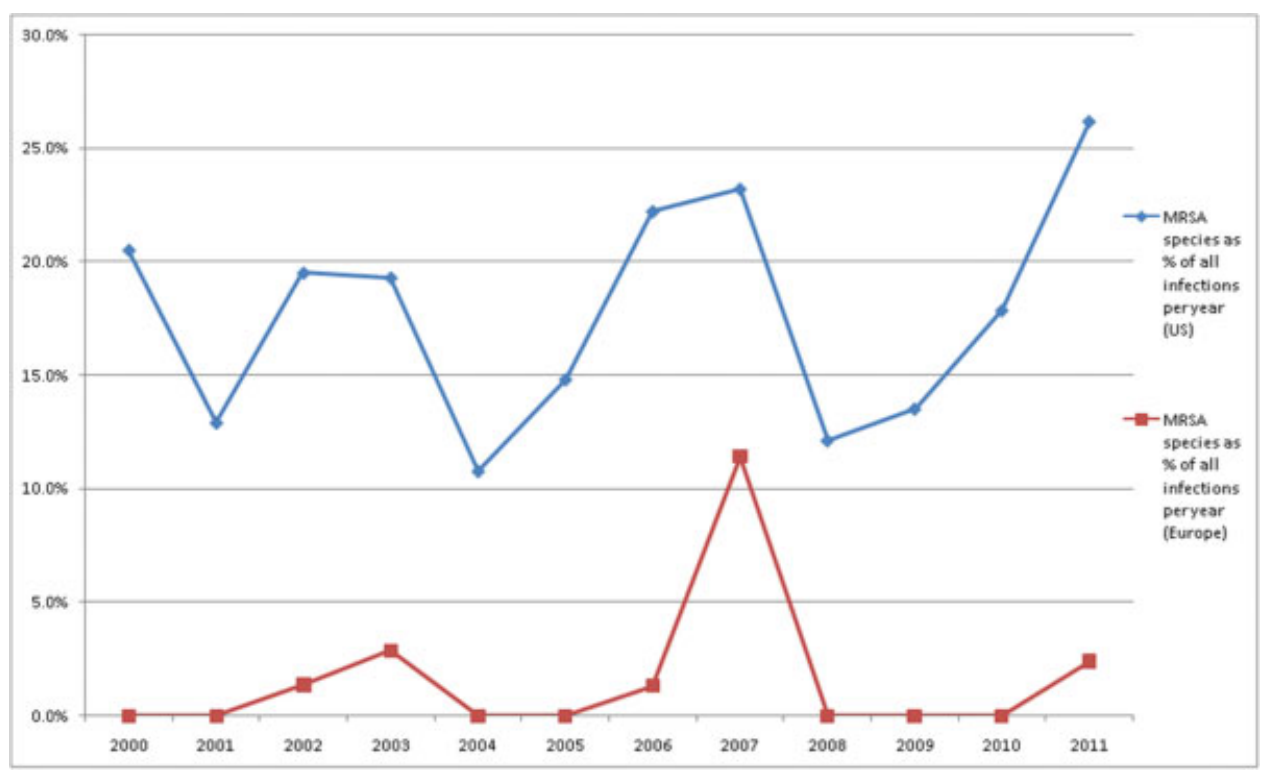

Fig. 4 Yearly trends of MRSA species (as percentage of total PJIs in given year) between United States and Europe.

step may be to determine what percentage of obese patients undergoing TJA are not receiving therapeutic doses of perioperative antibiotics during primary and revision surgery. ${ }^{32,33}$ With regard to infection control policies, the HELIOS ENDO-Klinik Hamburg in our study restricts use of vancomycin to only those cases with proven MRSA infection or colonization, while the use of vancomycin at the Rothman Institute may have been a bit more liberal, including patients with penicillin allergies. Furthermore, the HELIOS ENDOKlinik Hamburg bans use of scrubs outside of the operating room and disallows footwear worn in the operating room to be worn outside of the operating room -practices which were not routine at the Rothman Institute. Northern European nations such as the Netherlands, which have some of the world's lowest emergence of resistant organisms, also attribute their resistance rates to comprehensive infection control policy and restrictive use of antibiotics. ${ }^{28}$ With regard to the study design and methodology, only the difference in anaerobic species may be potentially attributable to the significant difference in mean culture incubation time, as the longer the incubation, the higher the percentage of indolent anaerobe grown.

The difference in organism profile between reconstruction centers in Europe and in the United States has significant implications regarding patient treatment methods and infection eradication. With the emergence of highly virulent and resistant strains of bacterial pathogens, surgeons in the United States have been forced to use high potency antibiotics such as vancomycin in an increasing number of patients. $^{10,14,34}$ This may lead to development of even more difficult to treat PJI given the limited availability of antibiotics used to eradicate new organisms such as vancomycin-

Table 1 Knee and hip data for the United States center and European center

\begin{tabular}{|l|l|l|l|l|}
\hline & United States & Europe & Hips (\%) \\
\hline Type of organism & Knees (\%) & Hips (\%) & Knees (\%) & 13.6 \\
\hline Staphylococcus aureus & 29.6 & 32.6 & 12.1 & 40.7 \\
\hline Coagulase-negative Staphylococcus & 21.7 & 18.4 & 37.0 & 12.9 \\
\hline Streptococcus and Enterococcus & 10.3 & 9.1 & 14.5 & 4.2 \\
\hline Gram negative & 6.4 & 6.8 & 4.5 & 3.5 \\
\hline Polymicrobial & 7.4 & 7.4 & 3.3 & 12.9 \\
\hline Anaerobe & 0.5 & 1.4 & 2.4 & 0.4 \\
\hline Fungal & 2.9 & 1.7 & 0.3 & 0.0 \\
\hline Mycobacterial & 0.5 & 0.8 & 0.0 & 1.1 \\
\hline Other & 4.5 & 6.5 & 0.6 & 10.9 \\
\hline Culture negative & 16.2 & 15.3 & 25.2 & \\
\hline
\end{tabular}


Table 2 Organism profiles for PJI reported in current study and selected existing literature

\begin{tabular}{|l|l|l|l|l|}
\hline & $\begin{array}{l}\text { Number of } \\
\text { cases analyzed }\end{array}$ & $\begin{array}{l}\text { Staphylococcus } \\
\text { aureus (\%) }\end{array}$ & $\begin{array}{l}\text { Coagulase-negative } \\
\text { Staphylococcus (\%) }\end{array}$ & Streptococcus (\%) \\
\hline Data from the United States & 772 & 31.0 & 20.2 & 9.7 \\
\hline Current study & 105 & 18.1 & 28.6 & 18.1 \\
\hline Fitzgerald 1995 & 146 & 35.0 & 31.0 & 11.0 \\
\hline Fulkerson et al 2006 & 55 & 43.6 & 20.0 & 10.9 \\
\hline Schinsky et al 2008 & \multicolumn{5}{l|}{} \\
\hline Data from Europe & 898 & 13.0 & 39.3 & 13.5 \\
\hline Current study & 426 & 33.6 & 31.0 & 18.4 \\
\hline Stefánsdóttir et al 2009 & 74 & 25.0 & 36.0 & 16.0 \\
\hline Phillips et al 2006 &
\end{tabular}

Abbreviation: PJI, periprosthetic joint infection.

resistant enterococcus. ${ }^{35}$ With regard to differences in surgical management, studies in both geographic regions have agreed that two-stage exchange arthroplasty is a successful procedure with eradication rates ranging from 82 to 100\%. ${ }^{21,36,37}$ European literature, however, also promotes the conservative single procedure approach for infection eradication: Romano et al cite an $81.9 \%$ success rate at an average 40.7 months follow-up, ${ }^{38}$ whereas Klouche et al even report a $100 \%$ success rate in 38 patients at minimum 2 years follow-up. In the United States on the other hand, there remains a significant hesitancy to perform one-stage direct exchange procedures, especially in situations where the infecting organism is $S$. aureus. ${ }^{39}$ Given the results of our study, future studies may need to examine the link between the greater number of $S$. aureus and antibiotic resistant PJIs in the United States and the caution of poor outcomes with direct prosthesis exchange in this region.

Our study is inherently limited by its retrospective design, which interferes with a consistent method of obtaining and analyzing cultures at each of the institutions. However, at both centers included in the study, standard institutional protocols for PJI were followed including obtaining at minimum three tissue cultures during surgery and growing specimens on standard media for at least 5 days. Second, since both institutions included are tertiary infection referral centers, there are several preoperative variables that we were unable to assess for their effect on specific type of infecting organism including date of index procedure, revision versus primary arthroplasty, and history of preoperative antibiotic therapy administration. Nonetheless, because the centers are huge referral centers for infection, we had the advantage of reporting on an enormous number of PJI cases with regard to infecting pathogen, more so than any study we are aware of in the current literature. Each cohort was an accurate representation of the predominant patient populations undergoing TJA in their respective geographic location, including the differences in patient demographics. Finally, it is important to note that although the two institutions included are well representative of the patient populations in the geographical regions, the results from this study must be examined with care and may not precisely represent all possible centers in the United States or in Europe.

\section{Conclusion}

Findings from our study show that the infecting organisms in PJI differ between an orthopedic center in Europe and in the United States. Not only are higher virulence organisms more prevalent in the American institution but a significantly greater resistance profile was discovered in the United States as well. Further studies will be needed to determine if the bacterial profile responsible for PJI in the two regions may be related to the difference in preferred surgical algorithms and reported outcomes after treatment. In general, the organism profile of each location will continue to play a large role in infection prevention and therapy after TJA.

\section{References}

1 Bozic KJ, Kurtz SM, Lau E, et al. The epidemiology of revision total knee arthroplasty in the United States. Clin Orthop Relat Res 2010; 468(1):45-51

2 Della Valle CJ, Zuckerman JD, Di Cesare PE. Periprosthetic sepsis. Clin Orthop Relat Res 2004;(420):26-31

3 Phillips JE, Crane TP, Noy M, Elliott TS, Grimer RJ. The incidence of deep prosthetic infections in a specialist orthopaedic hospital: a 15-year prospective survey. J Bone Joint Surg $\mathrm{Br} 2006 ; 88(7)$ : 943-948

4 Shuman EK, Urquhart A, Malani PN. Management and prevention of prosthetic joint infection. Infect Dis Clin North Am 2012;26(1): 29-39

5 Kurtz SM, Lau E, Schmier J, Ong KL, Zhao K, Parvizi J. Infection burden for hip and knee arthroplasty in the United States. J Arthroplasty 2008;23(7):984-991

6 Parvizi J, Azzam K, Ghanem E, Austin MS, Rothman RH. Periprosthetic infection due to resistant staphylococci: serious problems on the horizon. Clin Orthop Relat Res 2009;467(7): 1732-1739

7 Parvizi J, Pawasarat IM, Azzam KA, Joshi A, Hansen EN, Bozic KJ Periprosthetic joint infection: the economic impact of methicillinresistant infections. J Arthroplasty 2010;25(6, Suppl):103-107

8 Zmistowski B, Fedorka CJ, Sheehan E, Deirmengian G, Austin MS, Parvizi J. Prosthetic joint infection caused by gram-negative organisms. J Arthroplasty 2011;26(6, Suppl):104-108 
9 Kurtz SM, Lau E, Watson H, Schmier JK, Parvizi J. Economic burden of periprosthetic joint infection in the United States. J Arthroplasty 2012;27(8, Suppl):61-65, e1

10 Moran E, Masters S, Berendt AR, McLardy-Smith P, Byren I, Atkins BL. Guiding empirical antibiotic therapy in orthopaedics: the microbiology of prosthetic joint infection managed by debridement, irrigation and prosthesis retention. J Infect 2007;55(1):1-7

11 Mortazavi SMJ, Vegari D, Ho A, Zmistowski B, Parvizi J. Two-stage exchange arthroplasty for infected total knee arthroplasty: predictors of failure. Clin Orthop Relat Res 2011;469(11):3049-3054

12 Odum SM, Fehring TK, Lombardi AV, et al; Periprosthetic Infection Consortium. Irrigation and debridement for periprosthetic infections: does the organism matter? J Arthroplasty 2011;26(6, Suppl):114-118

13 Koyonos L, Zmistowski B, Della Valle CJ, Parvizi J. Infection control rate of irrigation and débridement for periprosthetic joint infection. Clin Orthop Relat Res 2011;469(11):3043-3048

14 Patel A, Calfee RP, Plante M, Fischer SA, Arcand N, Born C. Methicillin-resistant Staphylococcus aureus in orthopaedic surgery. J Bone Joint Surg Br 2008;90(11):1401-1406

15 Uçkay I, Bernard L. Gram-negative versus gram-positive prosthetic joint infections. Clin Infect Dis 2010;50(5):795

16 American Association of Orthopaedic Surgeons. AAOS releases new statement on antibiotics after arthroplasty. May 2009. Available at: http://www.aaos.org/news/aaosnow/may09/cover2.asp

17 Peel TN, Cheng AC, Buising KL, Choong PFM. Microbiological aetiology, epidemiology, and clinical profile of prosthetic joint infections: are current antibiotic prophylaxis guidelines effective? Antimicrob Agents Chemother 2012;56(5):2386-2391

18 Fitzgerald RH Jr. Infected total hip arthroplasty: diagnosis and treatment. J Am Acad Orthop Surg 1995;3(5):249-262

19 Fulkerson E, Valle CJD, Wise B, Walsh M, Preston C, Di Cesare PE. Antibiotic susceptibility of bacteria infecting total joint arthroplasty sites. J Bone Joint Surg Am 2006;88(6):1231-1237

20 Laffer RR, Graber P, Ochsner PE, Zimmerli W. Outcome of prosthetic knee-associated infection: evaluation of 40 consecutive episodes at a single centre. Clin Microbiol Infect 2006;12(5):433-439

21 Parvizi J, Zmistowski B, Adeli B. Periprosthetic joint infection: treatment options. Orthopedics 2010;33(9):659

22 Klouche S, Leonard P, Zeller V, et al. Infected total hip arthroplasty revision: one- or two-stage procedure? Orthop Traumatol Surg Res 2012;98(2):144-150

23 Charnley J, Eftekhar N. Postoperative infection in total prosthetic replacement arthroplasty of the hip-joint. With special reference to the bacterial content of the air of the operating room. Br J Surg 1969;56(9):641-649

24 Schinsky MF, Della Valle CJ, Sporer SM, Paprosky WG. Perioperative testing for joint infection in patients undergoing revision total hip arthroplasty. J Bone Joint Surg Am 2008;90(9):1869-1875

25 Stefánsdóttir A, Johansson D, Knutson K, Lidgren L, Robertsson 0. Microbiology of the infected knee arthroplasty: report from the Swedish Knee Arthroplasty Register on 426 surgically revised cases. Scand J Infect Dis 2009;41(11-12):831-840
26 Gorwitz RJ, Kruszon-Moran D, McAllister SK, et al. Changes in the prevalence of nasal colonization with Staphylococcus aureus in the United States, 2001-2004. J Infect Dis 2008;197(9):1226-1234

27 Schwarzkopf R, Takemoto RC, Immerman I, Slover JD, Bosco JA. Prevalence of Staphylococcus aureus colonization in orthopaedic surgeons and their patients: a prospective cohort controlled study. J Bone Joint Surg Am 2010;92(9):1815-1819

28 Bode LGM, Wertheim HFL, Kluytmans JAJW, et al. Sustained low prevalence of meticillin-resistant Staphylococcus aureus upon admission to hospital in The Netherlands. J Hosp Infect 2011; 79(3):198-201

29 Olofsson M, Lindgren P-E, Ostgren CJ, Midlöv P, Mölstad S. Colonization with Staphylococcus aureus in Swedish nursing homes: a cross-sectional study. Scand J Infect Dis 2012;44(1):3-8

30 Diekema DJ, Pfaller MA, Schmitz FJ, et al; SENTRY Partcipants Group. Survey of infections due to Staphylococcus species: frequency of occurrence and antimicrobial susceptibility of isolates collected in the United States, Canada, Latin America, Europe, and the Western Pacific region for the SENTRY Antimicrobial Surveillance Program, 1997-1999. Clin Infect Dis 2001;32(Suppl 2):S114-S132

31 Falagas ME, Karageorgopoulos DE. Adjustment of dosing of antimicrobial agents for bodyweight in adults. Lancet 2010;375 (9710):248-251

32 Hall RG II, Payne KD, Bain AM, et al. Multicenter evaluation of vancomycin dosing: emphasis on obesity. Am J Med 2008;121(6): 515-518

33 Roberts JA, Kruger P, Paterson DL, Lipman J. Antibiotic resistancewhat's dosing got to do with it? Crit Care Med 2008;36(8): 2433-2440

34 Jones ME, Karlowsky JA, Draghi DC, Thornsberry C, Sahm DF, Nathwani D. Epidemiology and antibiotic susceptibility of bacteria causing skin and soft tissue infections in the USA and Europe: a guide to appropriate antimicrobial therapy. Int J Antimicrob Agents 2003;22(4):406-419

35 Vercillo M, Patzakis MJ, Holtom P, Zalavras CG. Linezolid in the treatment of implant-related chronic osteomyelitis. Clin Orthop Relat Res 2007;461:40-43

36 Engesæter LB, Dale H, Schrama JC, Hallan G, Lie SA. Surgical procedures in the treatment of 784 infected THAs reported to the Norwegian Arthroplasty Register. Acta Orthop 2011;82(5): 530-537

37 Jämsen E, Stogiannidis I, Malmivaara A, Pajamäki J, Puolakka T, Konttinen YT. Outcome of prosthesis exchange for infected knee arthroplasty: the effect of treatment approach. Acta Orthop 2009; 80(1):67-77

38 Romanò CL, Gala L, Logoluso N, Romanò D, Drago L. Two-stage revision of septic knee prosthesis with articulating knee spacers yields better infection eradication rate than one-stage or twostage revision with static spacers. Knee Surg Sports Traumatol Arthrosc 2012;20(12):2445-2453

39 Choi H-R, von Knoch F, Zurakowski D, Nelson SB, Malchau H. Can implant retention be recommended for treatment of infected TKA? Clin Orthop Relat Res 2011;469(4):961-969 